\title{
Central limit theorems for hyperbolic spaces and Jacobi processes on $[0, \infty[$
}

\author{
Michael Voit \\ Fakultät Mathematik, Technische Universität Dortmund \\ Vogelpothsweg 87, D-44221 Dortmund, Germany \\ e-mail: michael.voit@math.tu-dortmund.de
}

November 6, 2018

\begin{abstract}
We present a unified approach to a couple of central limit theorems for radial random walks on hyperbolic spaces and time-homogeneous Markov chains on $[0, \infty[$ whose transition probabilities are defined in terms of the Jacobi convolutions. The proofs of all results are based on limit results for the associated Jacobi functions. In particular, we consider $\alpha \rightarrow \infty$, the case $\varphi_{i \rho-\lambda}^{(\alpha, \beta)}(t)$ for small $\lambda$, and $\varphi_{i \rho-n \lambda}^{(\alpha, \beta)}(t / n)$ for $n \rightarrow \infty$. The proofs of all these limit results are based on the known Laplace integral representation for Jacobi functions. Parts of the results are known, other improve known ones, and other are new.
\end{abstract}

KEYWORDS: Laplace integral representation, limits of Jacobi functions, asymptotic results, spherical functions, hyperbolic spaces, radial random walks, central limit theorems, normal limits, Rayleigh distributions.

Mathematics Subject Classification 2010: 60F05; 60B15; 33C45; 43A90; 43A62; 41A80.

\section{Introduction}

We here derive a couple of central limit theorems for the distance of radial random walks $\left(S_{n}^{k}\right)_{n \geq 0}$ from their starting points on the hyperbolic spaces $H_{k}(\mathbb{F})$ of dimension $k \geq 2$ over the fields $\mathbb{F}=\mathbb{R}, \mathbb{C}$, or the quaternions $\mathbb{H}$. The main observation for proofs is that the distance processes are again Markov chains on $[0, \infty[$ whose transition probabilities are related to to the product formula for the spherical functions for $H_{k}(\mathbb{F})$, i.e., certain Jacobi functions. As all proofs work without additional effort for more general "Jacobi random walks" on $[0, \infty[$, i.e., Markov processes on $[0, \infty[$ whose transition probabilities are related to general Jacobi functions, we shall derive all results in this context.

To describe the main results, we regard $H_{k}(\mathbb{F})$ as symmetric space $G / K$ with

$$
\begin{array}{ll}
\mathbb{F}=\mathbb{R}: & G=S O_{o}(1, k), \quad K=S O(k) \\
\mathbb{F}=\mathbb{C}: & G=S U(1, k), \quad K=S(U(1) \times U(k)) \\
\mathbb{F}=\mathbb{H}: & G=S p(1, k), \quad K=S p(1) \times S p(k)
\end{array}
$$

and define the dimension parameter $d:=\operatorname{dim}_{\mathbb{R}} \mathbb{F}=1,2,4$. We identify the double coset space 
$G / / K$ with the interval $[0, \infty[$ such that $t \in[0, \infty[$ corresponds with the double coset

$$
K a_{t} K \quad \text { with } \quad a_{t}=\left(\begin{array}{ccccc}
\operatorname{ch} t & 0 & \ldots & 0 & \operatorname{sh} t \\
0 & & & & 0 \\
\vdots & & I_{k-1} & & \vdots \\
0 & & & & 0 \\
\operatorname{sh} t & 0 & \ldots & 0 & \operatorname{ch} t
\end{array}\right)
$$

see e.g. [F] or [Hel]. Using this homeomorphism $\varphi: G / / K \rightarrow[0, \infty[$, we define the hyperbolic distance on $G / K$ by $d(x K, y K)=\varphi\left(K y^{-1} x K\right)$. In this way, $G$ acts on $G / K=H_{k}(\mathbb{F})$ isometrically in a two-point homogeneous way, i.e., for $x_{1}, x_{2}, y_{1}, y_{2} \in H_{k}(\mathbb{F})$ with $d\left(x_{1}, x_{2}\right)=$ $d\left(y_{1}, y_{2}\right)$ there exists $g \in G$ with $g\left(x_{1}\right)=y_{1}$ and $g\left(x_{2}\right)=y_{2}$.

Now consider a time-homogeneous Markov chain $\left(S_{n}^{k}\right)_{n \geq 0}$ on $H_{k}(\mathbb{F})$ with transition kernel $K_{k}$ starting at time 0 in $e K \in G / K=H_{k}(\mathbb{F})$. This Markov chain is called a radial random walk on $H_{k}(\mathbb{F})$, if $K_{k}$ is $G$-invariant, i.e., if for all $g \in G, x \in H_{k}(\mathbb{F})$, and Borel sets $A \subset H_{k}(\mathbb{F})$, $K_{k}(g(x), g(A))=K_{k}(g, A)$. It is well-known (see e.g. Lemma 4.4 of [RV1]) that then for the canonical projection $\pi: G / H=H_{k}(\mathbb{F}) \rightarrow G / / H=\left[0, \infty\left[\right.\right.$, the image process $\left(\pi\left(S_{n}^{k}\right)\right)_{n \geq 0}$ is a time-homogeneous Markov chain on $[0, \infty[$ with kernel

$$
\tilde{K}_{k}(x, A)=\left(\nu * \delta_{x}\right)(A) \quad \text { for } \quad x \in[0, \infty[\text { and Borel sets } A \subset[0, \infty[\text {, }
$$

where $\nu \in M^{1}\left(\left[0, \infty[)\right.\right.$ is the distribution of $d\left(S_{n}^{k}, S_{n+1}^{k}\right)$ (which is independent of $\left.n\right), \delta_{x}$ is a point measure, and $*$ denotes the the double coset convolution on $G / / H=[0, \infty[$. We point out that the Markov kernel $K_{k}$ and thus the finite-dimensional distributions of $\left(S_{n}^{k}\right)_{n \geq 0}$ are determined uniquely by $\nu \in M^{1}\left(\left[0, \infty[)\right.\right.$, and that the distributions of the distances $d\left(S_{n}^{\bar{k}}, e K\right)$ from the starting point $e K$ are the $n$-th convolution powers $\nu^{(n)}$ of $\nu$ w.r.t. *.

We are now interested in central limit theorems (CLTs) for $d\left(S_{n}^{k}, e K\right)$ for $n \rightarrow \infty$.

For the first type of results, we fix $\nu \in M^{1}\left(\left[0, \infty[)\right.\right.$ and $H_{k}(\mathbb{F})$, and introduce for each $c \in] 0,1]$ the compressing map $D_{c}: x \mapsto c x$ on $\left[0, \infty\left[\right.\right.$ as well as the compressed measure $\nu_{c}:=$ $D_{c}(\nu) \in M^{1}\left(\left[0, \infty[)\right.\right.$. Now consider the radial random walk $\left(S_{n}^{(k, c)}\right)_{n \geq 0}$ on $H_{k}(\mathbb{F})$ associated with the compressed measure $\nu_{c}$. We now look for CLTs for $\left(S_{n}^{\left(k, n^{-r}\right)}\right)_{n \geq 0}$ depending on $r \geq 0$ which include or supplement the results in [KTS], [Tu], [Gr], Section 3.2 of [Te], [Tr], and in $[\mathrm{Z} 1]$, [Z2], and Section 7.4 of the monograph [BH] in our setting.

The most classical case appears for $r=1 / 2$ and is well-known; see Theorem 7.4.1 of [BH] and references cited there. Here, the distance processes $\frac{1}{\sqrt{n}} d\left(S_{n}^{\left(k, n^{-r}\right)}, e K\right)$ from the starting point tend in distribution to some known limit depending on $H_{k}(\mathbb{F})$ and the second moment of $\nu$. These limit distributions are known as (radial parts) of Gaussians on $H_{k}(\mathbb{F})$. As this case is studied precisely in the literature, we omit this case here.

The case $r=0$ without initial compression is due to [Z1]; see Section 7.4 of [BH]. We here improve this CLT by a rate of convergence. The CLT needs the modified moment functions

$$
m_{j}(t):=m_{j}(d, k ; t):=\int_{0}^{1} \int_{0}^{\pi}\left(\ln \left(\left|\operatorname{ch} t+r \cdot e^{i \varphi} \operatorname{sh} t\right|\right)\right)^{j} d m_{d k / 2-1, d / 2-1}(r, \varphi) \quad(j \in \mathbb{N})
$$

with the probability measure $m_{d k / 2-1, d / 2-1} \in M^{1}([0,1] \times[0, \pi])$ defined below in (1.4). It will turn out in (2.4) below that this definition agrees with that in [Z1] and [BH]. It is known that $m_{1} \geq 0$ and $m_{1}(x)^{2} \leq m_{2}(x) \leq x^{2}$ for $x \geq 0$ with equality precisely for $x=0$. 
1.1 Theorem. Let $\nu \in M^{1}\left(\left[0, \infty[)\right.\right.$ with $\nu \neq \delta_{0}$ and finite second moment. For $j \in \mathbb{N}$ let $M_{j}:=\int_{0}^{\infty} m_{j}(t) d \nu(t)$ be the modified moments of $\nu$ with $M_{1}, M_{2}<\infty$ by our assumption. Then

$$
\frac{d\left(S_{n}^{(k, 1)}, e K\right)-n M_{1}}{\sqrt{n}}
$$

tends in distribution to $N\left(0, M_{2}-M_{1}^{2}\right)$ with $M_{2}-M_{1}^{2}>0$.

Moreover, if $\nu \in M^{1}([0, \infty[)$ in addition has a finite third moment, then the distribution functions of the random variables above tend uniformly on $\mathbb{R}$ to the distribution function of $N\left(0, M_{2}-M_{1}^{2}\right)$ of order $n^{-1 / 3}$.

The case $r>1 / 2$ was studied in [V2]. We reprove the result here in a different way:

1.2 Theorem. Let $\nu \in M^{1}\left(\left[0, \infty[)\right.\right.$ with $\nu \neq \delta_{0}$ and finite second moment $m_{2}:=\int_{0}^{\infty} x^{2} d \mu(x)$ (which is automatically positive). Then

$$
\left(\frac{d k}{m_{2}}\right)^{1 / 2} \cdot n^{r-1 / 2} \cdot d\left(S_{n}^{\left(k, n^{-r}\right)}, e K\right)
$$

tends in distribution to the Rayleigh distribution $\rho_{d k / 2-1}$, where $\rho_{\alpha}$ has the Lebesgue density

$$
\frac{1}{2^{\alpha} \Gamma(\alpha+1)} x^{2 \alpha+1} e^{-x^{2} / 2} \quad(x \geq 0) .
$$

Notice that in the preceding result $\operatorname{dim}_{\mathbb{R}} H_{k}(\mathbb{F})=d k$ and that $\rho_{d k / 2-1}$ is precisely the radial part of a $d k$-dimensional standard normal distribution on $\mathbb{R}^{d k}$. The preceding theorem therefore means that for $r>1 / 2$, the CLT forgets the curvature and admits the behavior of classical sums of i.i.d. random variables on the tangent space.

The case $r \in] 0,1 / 2[$ was not considered before. We here obtain:

1.3 Theorem. Let $\nu \in M^{1}\left(\left[0, \infty[)\right.\right.$ with $\nu \neq \delta_{0}$, with compact support, and thus with finite moments $\left.m_{l}:=\int_{0}^{\infty} x^{l} d \mu(x) \in\right] 0, \infty[(l \in \mathbb{N})$. Then:

(1) For $r \in] 1 / 6,1 / 2[$,

$$
\frac{d\left(S_{n}^{\left(k, n^{-r}\right)}, e K\right)-\frac{(d(k+1) / 2-1) m_{2} n^{1-2 r}}{d k}}{n^{1 / 2-r}}
$$

tends in distribution to $N\left(0, \frac{m_{2}}{d k}\right)$.

(2) If $r=1 / 6$, then the random variables of part (1) tend in distribution to $N\left(-M, \frac{m_{2}}{d k}\right)$ with the mean

$$
M:=-\frac{(d(k+1) / 2-1) d(k+3) / 2-2) \cdot m_{4}}{6 d k(d k / 2+1)} .
$$

(3) If $r \in] 0,1 / 6[$, then

$$
\frac{d\left(S_{n}^{\left.k, n^{-r}\right)}, e K\right)-\frac{(d(k+1) / 2-1) m_{2}}{d k} \cdot n^{1-2 r}}{n^{1-4 r}} \longrightarrow M
$$

in probability with $M$ as in (2).

Besides the preceding limit theorems for a fixed hyperbolic space, we also derive the following CLT for a fixed field $\mathbb{F}$, where the dimension $k$ and the number $n$ of steps tend to infinity. It generalizes a result in [V3]: 
1.4 Theorem. Let $\left(k_{n}\right)_{n \geq 1} \subset \mathbb{N}$ be increasing with $\lim _{n \rightarrow \infty} n / k_{n}=0$, and fix $\mathbb{F}$ as above. Let $\nu \in M^{1}\left(\left[0, \infty[)\right.\right.$ with finite second moment $\int_{0}^{\infty} x^{2} d \nu(x)$, and consider the associated radial random walks $\left(S_{n}^{k}\right)_{n \geq 0}$ on $H_{k}(\mathbb{F})$ for $k \in \mathbb{N}$. Then, $m_{j}:=\int_{0}^{\infty}(\ln (\operatorname{ch} x)) d \nu(x)<\infty$ exist for $j=1,2$, and

$$
\frac{d\left(S_{n}^{k_{n}}, S_{0}^{k_{n}}\right)-n m_{1}}{\sqrt{n}}
$$

tends in distribution for $n \rightarrow \infty$ to $N\left(0, m_{2}-m_{1}^{2}\right)$.

An extension of this CLT without the restriction $\lim _{n \rightarrow \infty} n / k_{n}=0$ was recently derived by Grundmann [G] by using completely different methods.

We now briefly describe the common roots of the proof of the preceding CLTs. We regard the spherical functions of the Gelfand pair $(G, H)$ above as continuous functions on $[0, \infty[$ which are multiplicative w.r.t. *, i.e., $f(x) f(y)=\int_{0}^{\infty} f d\left(\delta_{x} * \delta_{y}\right)$ for $x, y \geq 0$. It is well-known (see $[\mathrm{Ko} 2])$ that in our case all spherical functions are given by Jacobi functions

$$
\varphi_{\lambda}^{(\alpha, \beta)}(t):={ }_{2} F_{1}\left((\alpha+\beta+1-i \lambda) / 2,(\alpha+\beta+1+i \lambda) / 2 ; \alpha+1 ;-\operatorname{sh}^{2} t\right) \quad(\lambda \in \mathbb{C})
$$

with the parameters

$$
\alpha=d k / 2-1, \quad \beta=d / 2-1 \quad \text { with } \quad d:=\operatorname{dim}_{\mathbb{R}} \mathbb{F}=1,2,4 .
$$

Moreover, the double coset convolutions $*$ on $[0, \infty[$ for the hyperbolic spaces above can be regarded as special cases of Jacobi convolution $*_{(\alpha, \beta)}$ on $[0, \infty[$ which were investigated mainly by Flensted-Jensen and Koornwinder. In the following we refer to the survey [Ko2] on the subject. For $\alpha>\beta \geq-1 / 2$ with $\alpha>-1 / 2$, this convolution is given by

$$
\delta_{s} *(\alpha, \beta) \delta_{t}(f):=\int_{0}^{1} \int_{0}^{\pi} f\left(\operatorname{arch}\left|\operatorname{ch} s \cdot \operatorname{ch} t+r e^{i \varphi} \operatorname{sh} s \cdot \operatorname{sh} t\right|\right) d m_{\alpha, \beta}(r, \varphi)
$$

for $f \in C_{b}\left(\left[0, \infty[)\right.\right.$ and for the probability measure $d m_{\alpha, \beta}$ with

$$
d m_{\alpha, \beta}(r, \varphi)=\frac{2 \Gamma(\alpha+1)}{\Gamma(1 / 2) \Gamma(\alpha-\beta) \Gamma(\beta+1 / 2)} \cdot\left(1-r^{2}\right)^{\alpha-\beta-1}(r \sin \varphi)^{2 \beta} \cdot r d r d \varphi
$$

for $\alpha>\beta>-1 / 2$. For $\alpha>\beta=-1 / 2$, the measure degenerates into

$$
d m_{\alpha,-1 / 2}(r, \varphi)=\frac{2 \Gamma(\alpha+1)}{\Gamma(1 / 2) \Gamma(\alpha+1 / 2)}\left(1-r^{2}\right)^{\alpha-1 / 2} d r \cdot \frac{1}{2} d\left(\delta_{0}+\delta_{\pi}\right)(\varphi)
$$

and for $\alpha=\beta>-1 / 2$ into

$$
d m_{\alpha, \alpha}(r, \varphi)=\frac{2 \Gamma(\alpha+1)}{\Gamma(1 / 2) \Gamma(\alpha+1 / 2)} \sin ^{2 \alpha} \varphi d \varphi \cdot d \delta_{0}(r) .
$$

Now fix $\alpha \geq \beta \geq-1 / 2$ with $\alpha>-1 / 2$. It is well-known that the Jacobi convolution above can be extended uniquely in a weakly continuous, bilinear way to a probability-preserving convolution $*_{(\alpha, \beta)}$ on $M_{b}([0, \infty[)$, and that one obtains the so-called Jacobi-type hypergroups on $[0, \infty[$; see $[\mathrm{Ko} 2],[\mathrm{BH}],[\mathrm{Tr}]$.

Using this Jacobi-convolution, we now generalize the Markov processes $\left(d\left(S_{n}^{k}, e K\right)\right)_{n \geq 0}$ above as follows: Fix a measure $\nu \in M^{1}([0, \infty[)$, and consider a time-homogeneous Jacobi 
random walk $\left(S_{n}^{(\alpha, \beta)}\right)_{n \geq 0}$ on $[0, \infty[$ with law $\nu$ of index $(\alpha, \beta)$, i.e., a time-homogeneous Markov process on $[0, \infty[$ starting at 0 with transition probability

$$
P\left(S_{n+1}^{(\alpha, \beta)} \in A \mid S_{n}^{(\alpha, \beta)}=x\right)=\left(\delta_{x} *(\alpha, \beta) \nu\right)(A) \quad(x \geq 0, A \subset[0, \infty[\quad \text { a Borel set }) .
$$

This notion agrees with that for $\left(d\left(S_{n}^{k}, e K\right)\right)_{n \geq 0}$ in the hyperbolic case above. We shall derive all CLTs above in this more general setting. We shall do this in Section 3 for growing dimensions and in Section 4 for fixed dimension $k$. In this way, Theorems 1.2, 1.1, 1.3, and 1.4 are just special cases of Theorems 4.3, 4.2, 4.7 and 3.1 below respectively. The proofs of all these limit theorems will be be based on several limit results for Jacobi functions which we will derive in Section 2. The basis of all these limit results will be the following well-known Laplace integral representation for the Jacobi functions; see Section 5.2 of [Ko2]:

1.5 Theorem. Let $\alpha \geq \beta \geq-1 / 2$. Then, for $\lambda \in \mathbb{C}$ and $t \geq 0$,

$$
\varphi_{\lambda}^{(\alpha, \beta)}(t)=\int_{0}^{1} \int_{0}^{\pi}\left|\operatorname{ch} t+r e^{i \varphi} \operatorname{sh} t\right|^{i \lambda-\rho} d m_{\alpha, \beta}(r, \varphi)
$$

with

$$
\rho:=\alpha+\beta+1 \geq 0
$$

and the probability measure $d m_{\alpha, \beta}$ introduced in (1.4),(1.5) and (1.6) respectively.

We mention that Theorem 1.5 admits an analogue for Jacobi polynomials due to Koornwinder [Ko1], and that this integral representation also leads to limit results. In particular, one can revisit Hilb's formula for Jacobi polynomials (see [Sz]) in order to prove CLTs for Markov chains on $\mathbb{Z}_{+}$associated with orthogonal polynomials; see [Ga], [V1], Section 7.4 of $[\mathrm{BH}]$, and references cited there for the topic.

We also mention that parts of this paper can be extended to certain families of HeckmanOpdam hypergeometric functions of type BC which include the spherical functions for the symmetric spaces $S U(p, q) /(S U(p) \times S U(q))$. For the background on these functions and the associated convolution structures on Weyl chambers of type B we refer to $[\mathrm{H}],[\mathrm{HS}],[\mathrm{O}]$ and $[\mathrm{R}]$. In [RV2] we generalize the Harish Chandra integral representation for $S O_{0}(p, q) /(S O(p) \times$ $S O(q))$ for $\mathbb{F}=\mathbb{R}$ of $[\mathrm{Sa}]$ to the more general setting considered in $[\mathrm{R}]$; this integral representation is similar to that in Theorem 1.5 and leads with more technical effort to multidimensional extensions of some of the result in the present paper.

\section{Limit relations for Jacobi functions}

We start with two major results where $\alpha$ or both parameters $\alpha, \beta$ converge to infinity.

2.1 Proposition. Let $\beta \geq-1 / 2$. Then there exists a constant $C=C(\beta)$ such that for all $t \geq 0, \alpha>\max (\beta, 0)$, and $\lambda \in \mathbb{R}$

$$
\left|\varphi_{i \rho-\lambda}^{(\alpha, \beta)}(t)-e^{i \lambda \cdot \ln (\operatorname{ch} t)}\right| \leq C \frac{|\lambda| \cdot \min (1, t)}{\alpha^{1 / 2}} .
$$

Proof. Using Theorem 1.5, we consider the difference

$$
R:=\left|\varphi_{i \rho-\lambda}^{(\alpha, \beta)}(t)-e^{i \lambda \cdot \ln (\operatorname{ch} t)}\right|=\left|\int_{0}^{1} \int_{0}^{\pi}\left(\left|\operatorname{ch} t+r e^{i \varphi} \operatorname{sh} t\right|^{i \lambda}-|\operatorname{ch} t|^{i \lambda}\right) d m_{\alpha, \beta}(r, \varphi)\right|
$$


which satisfies

$$
R \leq \int_{0}^{1} \int_{0}^{\pi}\left|g\left(r e^{i \varphi}, t\right)-1\right| d m_{\alpha, \beta}(r, \varphi)
$$

with

$$
g\left(r e^{i \varphi}, t\right):=\left|1+r e^{i \varphi} \cdot \operatorname{sh} t / \operatorname{ch} t\right|^{i \lambda}=e^{i \lambda \cdot \ln \left(\left|1+r e^{i \varphi} \cdot \operatorname{sh} t / \operatorname{ch} t\right|\right)} .
$$

As $\left|e^{i x}-1\right| \leq \sqrt{2} \cdot|x|$ for $x \in \mathbb{R}$, we have

$$
\left|g\left(r e^{i \varphi}, t\right)-1\right| \leq \sqrt{2} \cdot|\lambda| \cdot\left|\ln \left(\left|1+r e^{i \varphi} \cdot \operatorname{sh} t / \operatorname{ch} t\right|\right)\right| .
$$

Moreover, as for $z \in \mathbb{C}$ with $|z|<1$

$$
|\ln (|1+z|)| \leq|\ln (1+z)| \leq|z|+|z|^{2}+|z|^{3} \ldots=|z| /(1-|z|),
$$

and as $0 \leq \operatorname{sh} t / \operatorname{ch} t \leq \min (1, t)$ for $t \geq 0$, we obtain for $0 \leq r \leq 1$ and $t \geq 0$

$$
\left|g\left(r e^{i \varphi}, t\right)-1\right| \leq \sqrt{2} \cdot|\lambda| \cdot \frac{r \cdot \operatorname{sh} t / \operatorname{ch} t}{1-r \cdot \operatorname{sh} t / \operatorname{ch} t} \leq \sqrt{2} \cdot|\lambda| \cdot \min (1, t) \frac{r}{1-r} .
$$

Therefore, as $r \in[0,1]$,

$$
\left|g\left(r e^{i \varphi}, t\right)-1\right| \leq 2 \sqrt{2} \cdot|\lambda| \cdot \min (1, t) \frac{r}{1-r^{2}} .
$$

Now consider the probability measure $m_{\alpha, \beta}$ for $\alpha>\beta>-1 / 2$ introduced in Theorem 1.5. We conclude that

$$
R \leq 2 \sqrt{2} \cdot|\lambda| \cdot \frac{2 \cdot \min (1, t) \cdot \Gamma(\alpha+1)}{\Gamma(1 / 2) \Gamma(\alpha-\beta) \Gamma(\beta+1 / 2)} \int_{0}^{\pi} \sin ^{2 \beta} \varphi d \varphi \cdot \int_{0}^{1}\left(1-r^{2}\right)^{\alpha-\beta-2} r^{2 \beta+2} d r .
$$

Using standard formulas for the beta-integrals on the right-hand side and finally

$$
\Gamma(\alpha+1) / \Gamma(\alpha+1 / 2)=O(\sqrt{\alpha}) \quad(\alpha \rightarrow \infty)
$$

we obtain

$$
R \leq 8 \cdot|\lambda| \cdot \min (1, t) \frac{\Gamma(\beta+3 / 2)}{\Gamma(\beta+1)} \cdot \frac{\Gamma(\alpha+1)}{(\alpha-\beta-1) \Gamma(\alpha+1 / 2)}=|\lambda| \cdot \min (1, t) \cdot O(1 / \sqrt{\alpha})
$$

as claimed. The case $\beta=-1 / 2$ follows in the same way from the definition of $m_{\alpha, \beta}$.

We now consider a variant where $\alpha$ and $\beta$ tend to infinity in a coupled way. We note that the convergence results 2.1 and 2.2 (without error estimates) correspond to well-known limit transitions for Jacobi polynomials which can be found e.g. in [Ko3] or [Sz].

2.2 Proposition. Fix constants $c>1$ and $d>0$ and put $\alpha:=c \beta+d$. Then there exists a constant $C=C(c, d)$ such that for all $t \geq 0, \beta>0$, and $\lambda \in \mathbb{R}$

$$
\left|\varphi_{i \rho-\lambda}^{(c \beta+d, \beta)}(t)-e^{i \lambda \cdot \ln \sqrt{\mathrm{ch}^{2} t-(1 / c) \operatorname{sh}^{2} t}}\right| \leq C \frac{|\lambda| \cdot \min (1, t)}{\beta^{1 / 2}} .
$$

The proof will be based on the following observation which is likely well-known. 
2.3 Lemma. Consider a continuous function $f:[0,1] \rightarrow\left[0, \infty\left[\right.\right.$ such that there exist $x_{0} \in$ $[0,1]$ and constants $0<c_{1} \leq c_{2} \leq 1$ such that $f(x) \leq 1-c_{2}\left(x-x_{0}\right)^{2}$ holds for all $x \in[0,1]$, and $f(x) \geq 1-c_{1}\left(x-x_{0}\right)^{2}$ for all $x \in[0,1]$ in a suitable neighborhood of $x_{0}$. Moreover, let $g:[0,1] \rightarrow\left[0, \infty\left[\right.\right.$ be continuous with $g\left(x_{0}\right)>0$. Then, for continuous $n \rightarrow \infty$,

$$
\int_{0}^{1}\left|x-x_{0}\right| \cdot f^{n}(x) \cdot g(x) d x=O\left(\frac{1}{\sqrt{n}} \int_{0}^{1} f^{n}(x) \cdot g(x) d x\right) .
$$

Proof. For $n$ sufficiently large, we obtain by continuity arguments and omitting parts of the left hand integral that

$$
\int_{0}^{1} f^{n}(x) \cdot g(x) d x \geq \int_{0}^{1 / \sqrt{c_{1} n}}\left(1-c_{1} x^{2}\right)^{n} g\left(x_{0}\right) / 2 d x=\frac{g\left(x_{0}\right)}{2 \sqrt{c_{1} n}} \int_{0}^{1}\left(1-y^{2} / n\right)^{n} d y
$$

where the integral on the right hand side converges to some positive constant. On the other hand,

$$
\begin{aligned}
\int_{0}^{1}\left|x-x_{0}\right| \cdot f^{n}(x) \cdot g(x) d x & =\left(\int_{0}^{x_{0}}+\int_{x_{0}}^{1}\right)\left|x-x_{0}\right| \cdot f^{n}(x) \cdot g(x) d x \\
& \leq 2 \int_{0}^{1} x\left(1-c_{2} x^{2}\right)^{n} \cdot h(x) d x \\
& =\frac{2}{n \sqrt{c_{2}}} \int_{0}^{\sqrt{c_{2} n}}\left(1-y^{2} / n\right)^{n} \cdot h\left(y / \sqrt{n c_{2}}\right) d y
\end{aligned}
$$

for some continuous function $h$ depending on $g$ where the integral on the right hand side converges to some finite positive constant. A combination of both results leads to the lemma.

Proof of Proposition 2.2. Precisely as in the proof of Proposition 2.1 we obtain that

$$
R:=\left|\varphi_{i \rho-\lambda}^{(\alpha, \beta)}(t)-e^{i \lambda \cdot \ln \sqrt{\operatorname{ch}^{2} t-(1 / c) \operatorname{sh}^{2} t}}\right| \leq 2|\lambda| \int_{0}^{1} \int_{0}^{\pi}|\ln A| d m_{\alpha, \beta}(r, \varphi)
$$

for

$$
A:=\frac{\left|\operatorname{ch} t+r e^{i \varphi} \operatorname{sh} t\right|}{\left|\operatorname{ch} t+i \cdot \frac{1}{\sqrt{c}} \operatorname{sh} t\right|}
$$

As for $r \in[0,1]$ and $c>1$, we have $(1-r) / 2 \leq A \leq 1+r$, we obtain

$$
|A-1| \leq \max (r,(r+1) / 2)=(r+1) / 2 .
$$

Therefore, using $|\ln (|1+z|)| \leq|z| /(1-|z|)$ for $|z|=|A-1| \leq 1$ as in the preceding proof, we conclude that

$$
|\ln A| \leq \frac{|A-1|}{1-|A-1|} \leq \frac{2}{1-r} \cdot \frac{\left|1-A^{2}\right|}{|1+A|} \leq \frac{2}{1-r} \cdot\left|1-A^{2}\right| \leq \frac{4}{1-r^{2}} \cdot\left|1-A^{2}\right| .
$$

Moreover, defining $\tau:=\operatorname{sh} t / \operatorname{ch} t \leq \min (t, 1)$, we have

$$
\begin{aligned}
\left|1-A^{2}\right| & =\left|1-\frac{(1+r \tau \cos \varphi)^{2}+r^{2} \tau^{2} \sin ^{2} \varphi}{1+\tau^{2} / c}\right|=\left|\frac{\tau^{2}\left(1 / c-r^{2}\right)+2 r \tau \cos \varphi}{1+\tau^{2} / c}\right| \\
& \leq \tau\left(2|\cos \varphi|+\left|\frac{\left|1 / c-r^{2}\right|}{1+\tau^{2} / c}\right|\right) \leq 2 \cdot \tau(|\cos \varphi|+|r-1 / \sqrt{c}|) .
\end{aligned}
$$


In summary, we have

$$
\begin{aligned}
R & \leq 16|\lambda| \tau \cdot \int_{0}^{1} \int_{0}^{\pi} \frac{|\cos \varphi|+|r-1 / \sqrt{c}|}{1-r^{2}} d m_{\alpha, \beta}(r, \varphi) \\
& =16|\lambda| \tau \cdot \frac{2 \Gamma(\alpha+1)}{\Gamma(1 / 2) \Gamma(\alpha-\beta) \Gamma(\beta+1 / 2)} \\
& \cdot \int_{0}^{1} \int_{0}^{\pi}(|\cos \varphi|+|r-1 / \sqrt{c}|) \cdot\left(\left(1-r^{2}\right) r^{2 /(c-1)}\right)^{\beta(c-1)-2} \cdot r^{1+4 /(c-1)}\left(1-r^{2}\right)^{d} \cdot \sin ^{2 \beta} \varphi d r d \varphi .
\end{aligned}
$$

We now apply Lemma 2.3 to $g(r):=r^{1+4 /(c-1)}\left(1-r^{2}\right)^{d}$ and $f(r):=\left(1-r^{2}\right) r^{2 /(c-1)}$ with the maximum value of $f$ on $[0,1]$ at $r_{0}=1 / \sqrt{c}$ and notice that $d m_{\alpha, \beta}(r, \varphi)$ is a probability measure. This yields

$$
\left.\int_{0}^{1}|r-1 / \sqrt{c}|\right) \cdot\left(\left(1-r^{2}\right) r^{2 /(c-1)}\right)^{\beta(c-1)-2} \cdot r^{1+4 /(c-1)}\left(1-r^{2}\right)^{d} d r=O(1 / \sqrt{\beta}) .
$$

As a similar argument also yields

$$
\int_{0}^{\pi}|\cos \varphi| \cdot \sin ^{2 \beta} \varphi d \varphi=O(1 / \sqrt{\beta})
$$

we obtain $R \leq 16|\lambda| \tau \cdot O(1 / \sqrt{\beta})$ as claimed.

We next turn to a limit concerning Bessel functions. Recapitulate that the normalized Bessel functions

$$
j_{\alpha}(t):={ }_{0} F_{1}\left(\alpha+1 ;-t^{2} / 4\right)=\Gamma(\alpha+1) \cdot \sum_{n=0}^{\infty} \frac{(-1)^{n}(t / 2)^{2 n}}{n ! \Gamma(n+\alpha+1)}
$$

with $j_{\alpha}(0)=1$ for $\alpha>-1 / 2$ admit the integral representation

$$
j_{\alpha}(t)=\frac{\Gamma(\alpha+1)}{\Gamma(1 / 2) \Gamma(\alpha+1 / 2)} \int_{-1}^{1} e^{i t u}\left(1-u^{2}\right)^{\alpha-1 / 2} d u .
$$

In order to compare $j_{\alpha}$ with the Jacobi functions, we rewrite it as

$$
j_{\alpha}(t)=\int_{0}^{1} \int_{0}^{\pi} e^{i t r \cos \varphi} d m_{\alpha, \beta}(r, \varphi)
$$

for $\alpha>\beta \geq-1 / 2$. In fact, the right hand side of (2.3) can be easily reduced to (2.2) by applying first polar coordinates $u=r \cos \varphi, v=r \sin \varphi$ with $v \in\left[0, \sqrt{1-u^{2}}\right]$ and then the transform $z:=v / \sqrt{1-u^{2}} \in[0,1]$, where the $z$-integral fits the constants.

The following limit result is related to the asymptotic expansion of the Jacobi functions in terms of Bessel functions in [ST]; see Lemma 3.3 in [V2].

2.4 Proposition. Let $\alpha \geq \beta \geq-1 / 2$ with $\alpha>-1 / 2$ and $T>0$ a constant. There exists a constant $C=C(\alpha, \beta, T)$ such that for all $\lambda \in \mathbb{R}, t \in[0, T]$, and $n \geq 1$,

$$
\left|\varphi_{i \rho-n \lambda}^{(\alpha, \beta)}(t / n)-j_{\alpha}(\lambda t)\right| \leq C \cdot|\lambda| t^{2} / n .
$$


Proof. The integral representations in Theorem 1.5 and (2.3) imply that

$$
\begin{aligned}
R & :=\left|\varphi_{i \rho-n \lambda}^{(\alpha, \beta)}(t / n)-j_{\alpha}(\lambda t)\right| \\
& \leq \int_{0}^{1} \int_{0}^{\pi}\left|\exp \left(i \lambda n \cdot \ln \left|\operatorname{ch}(t / n)+r e^{i \varphi} \operatorname{sh}(t / n)\right|\right)-e^{i \lambda t r \cos \varphi}\right| d m_{\alpha, \beta}(r, \varphi) .
\end{aligned}
$$

Using the well-known inequality $\left|e^{i x}-e^{i y}\right| \leq \sqrt{2} \cdot|x-y|$ for $x, y \in \mathbb{R}$, we obtain

$$
R \leq|\lambda| \cdot \int_{0}^{1} \int_{0}^{\pi}|n \cdot \ln | \operatorname{ch}(t / n)+r e^{i \varphi} \operatorname{sh}(t / n)|-\operatorname{tr} \cos \varphi| d m_{\alpha, \beta}(r, \varphi) .
$$

As

$$
\begin{aligned}
\ln \left|\operatorname{ch}(t / n)+r e^{i \varphi} \operatorname{sh}(t / n)\right| & =\frac{1}{2} \ln \left((\operatorname{ch}(t / n)+r \cos \varphi \operatorname{sh}(t / n))^{2}+r^{2} \sin ^{2} \varphi \operatorname{sh}^{2}(t / n)\right. \\
& =\frac{1}{2} \ln \left(1+2 r \cos \varphi \cdot t / n+t^{2}\left(1+r^{2}\right) / n^{2}+O\left(t^{3} / n^{3}\right)\right) \\
& =\frac{1}{2}\left(2 r \cos \varphi \frac{t}{n}+\frac{t^{2}\left(1+r^{2}\right)}{n^{2}}-2 r^{2} \cos ^{2} \varphi \frac{t^{2}}{n^{2}}+O\left(\frac{t^{3}}{n^{3}}\right)\right)
\end{aligned}
$$

uniformly for $t \in[0, T], r \in[0,1], \varphi \in[0, \pi]$, the claim follows.

The next result describes the oscillatory behavior of $\varphi_{i \rho-\lambda}^{(\alpha, \beta)}(t)$ in the spectral variable $\lambda \in \mathbb{R}$ for fixed $\alpha, \beta$, which is uniform in $t \geq 0$. For this we follow Section 7.2.2 of [BH] and define for $k \in \mathbb{N}$ the so called moment functions

$$
\begin{aligned}
m_{k}(t):=m_{k}^{(\alpha, \beta)}(t) & :=\left.\frac{\partial^{k}}{\partial \lambda^{k}} \varphi_{i \rho+i \lambda}^{(\alpha, \beta)}(t)\right|_{\lambda=0}=\left.\frac{\partial^{k}}{\partial \lambda^{k}} \varphi_{-i \rho-i \lambda}^{(\alpha, \beta)}(t)\right|_{\lambda=0} \\
& =\int_{0}^{1} \int_{0}^{\pi}\left(\ln \left(\left|\operatorname{ch} t+r \cdot e^{i \varphi} \operatorname{sh} t\right|\right)\right)^{k} d m_{\alpha, \beta}(r, \varphi)
\end{aligned}
$$

for $t \geq 0$ (where the second last equation follows from symmetry of the Jacobi functions in the parameter, and last one from Theorem 1.5). In particular, the function $m_{1}$ appears as substitute of the additive function $x \mapsto x$ on the group $(\mathbb{R},+)$ on the Jacobi convolution structure $\left(\left[0, \infty\left[, *_{\alpha, \beta}\right)\right.\right.$; see $[\mathrm{Z} 1]$ and Section 7.2 of the monograph $[\mathrm{BH}] . m_{1}$ can be used to define a modified drift-part in a CLT on $[0, \infty[$; see $[\mathrm{Z1}][\mathrm{BH}]$ and Section 3 below. We mention that for the parameters $\alpha, \beta$, for which the Jacobi functions are spherical functions of rank-one, non-compact symmetric spaces, this meaning of $m_{1}$ is well-known for a long time in probability theory on on hyperbolic spaces; see, for instance, [KTS], [Tu], [F].

We now derive a result which improves a general result of Zeuner [Z1] for general ChebliTrimeche hypergroups on $[0, \infty]$ in the special case of the Jacobi convolution structures $\left(\left[0, \infty\left[, *_{\alpha, \beta}\right)\right.\right.$. It will be used to derive a Berry-Esseen-type CLT below.

2.5 Proposition. Let $\alpha \geq \beta \geq-1 / 2$ with $\alpha>-1 / 2$. Then there exists a constant $C=$ $C(\alpha, \beta)$ such that for all $t \geq 0$ and $\lambda \in \mathbb{R}$,

$$
\left|\varphi_{i \rho-\lambda}^{(\alpha, \beta)}(t)-e^{i \lambda \cdot m_{1}(t)}\right| \leq C\left(\lambda^{2}+|\lambda|^{3}\right) .
$$

The proof depends on the following elementary observation: 
2.6 Lemma. For $z \in \mathbb{C}$ with $|z| \leq 1, \varepsilon \in] 0,1]$, and the Euler number $e=2,71 \ldots$,

$$
|\ln | 1+z|| \leq \frac{1}{e \varepsilon(1-|z|)^{\varepsilon}}
$$

Proof. Elementary calculus yields $\left|x^{\varepsilon} \cdot \ln x\right| \leq 1 /(e \varepsilon)$ for $\left.\left.x \in\right] 0,1\right]$. Therefore,

$$
\begin{aligned}
|\ln | 1+z|| & =|\Re \ln (1+z)| \leq|\ln (1+z)|=\left|z-z^{2} / 2+z^{3} / 3 \pm \ldots\right| \\
& \leq|z|+|z|^{2} / 2+|z|^{3} / 3 \pm \ldots=|\ln (1-|z|)| \leq \frac{1}{e \varepsilon(1-|z|)^{\varepsilon}} .
\end{aligned}
$$

Proof of the Proposition: Let $h(t, r, \varphi):=\left|1+r e^{i \varphi} \cdot \operatorname{sh} t / \operatorname{ch} t\right|$. Then, for $t \geq 0$,

$$
e^{i \lambda \cdot m_{1}(t)}=(\operatorname{ch} t)^{i \lambda} \cdot \exp \left(i \lambda \int_{0}^{1} \int_{0}^{\pi} \ln (h(t, r, \varphi)) d m_{\alpha, \beta}(r, \varphi)\right) .
$$

Therefore, using the integral representation of the Jacobi functions, we obtain

$$
\begin{aligned}
R & :=\left|\varphi_{i \rho-\lambda}^{(\alpha, \beta)}(t)-e^{i \lambda \cdot m_{1}(t)}\right| \\
& =\left|\int_{0}^{1} \int_{0}^{\pi} e^{i \lambda \cdot \ln (h(t, r, \varphi))} d m_{\alpha, \beta}(r, \varphi)-\exp \left(i \lambda \int_{0}^{1} \int_{0}^{\pi} \ln (h(t, r, \varphi)) d m_{\alpha, \beta}(r, \varphi)\right)\right| .
\end{aligned}
$$

We now write down the usual power series for both exponentials and observe that the terms of order 0 and 1 are equal in both expansions. Therefore,

$$
\begin{aligned}
R \leq \int_{0}^{1} & \int_{0}^{\pi}\left|e^{i \lambda \cdot \ln (h(t, r, \varphi))}-(1+i \lambda \cdot \ln (h(t, r, \varphi)))\right| d m_{\alpha, \beta}(r, \varphi) \\
& +\left|\exp \left(i \lambda \int_{0}^{1} \int_{0}^{\pi} \ln (h(t, r, \varphi)) d m_{\alpha, \beta}(r, \varphi)\right)-1-i \lambda \int_{0}^{1} \int_{0}^{\pi} \ln (h(t, r, \varphi)) d m_{\alpha, \beta}(r, \varphi)\right| .
\end{aligned}
$$

Using the well-known estimates $|\cos x-1| \leq x / 2$ and $|\sin x-x| \leq|x|^{3} / 6$ for $x \in \mathbb{R}$, we obtain $\left|e^{i x}-(1+i x)\right| \leq x / 2+|x|^{3} / 6$, and thus

$$
R \leq A_{1}^{2} \lambda^{2} / 2+A_{1}^{3}|\lambda|^{3} / 6+A_{2} \lambda^{2} / 2+A_{3}|\lambda|^{3} / 6
$$

for $A_{k}:=\int_{0}^{1} \int_{0}^{\pi}|\ln (h(t, r, \varphi))|^{k} d m_{\alpha, \beta}(r, \varphi), k=1,2,3$. In particular, by Jensen's inequality,

$$
R \leq A_{2} \lambda^{2}+A_{3}|\lambda|^{3} / 3 \text {. }
$$

Assume now that $\alpha>\beta>-1 / 2$ holds. Choose some $\varepsilon \in] 0,1[$ with $\varepsilon<(\alpha-\beta) / 3$ and apply Lemma 2.6 as well as $1-r \operatorname{sh} t / \operatorname{ch} t \geq 1-r \geq(1-r) / 2$ for $r \in[0,1], t \geq 0$. This and the definitions of $A_{k}$ and $h$ imply for $k=2,3$ and some constants $C_{1}, C_{2}, C_{3}$ that

$$
\begin{aligned}
A_{k} & \leq \int_{0}^{1} \int_{0}^{\pi} \frac{C_{1}}{\cdot|1-r \operatorname{sh} t / \operatorname{ch} t|^{k \varepsilon}} d m_{\alpha, \beta}(r, \varphi) \\
& \leq C_{2} \int_{0}^{1} \int_{0}^{\pi}(1-r)^{\alpha-\beta-1-k \varepsilon}(r \sin \varphi)^{2 \beta} \cdot r d r d \varphi \leq C_{3}
\end{aligned}
$$

as claimed.

The case $\alpha>\beta=-1 / 2$ follows in the same way by using $m_{\alpha,-1 / 2}$.

Finally, the case $\alpha=\beta>-1 / 2$ can be reduced to the case $\alpha>\beta=-1 / 2$ by the well-known quadratic transform $\varphi_{2 \lambda}^{(\alpha, \alpha)}(t)=\varphi_{\lambda}^{(\alpha,-1 / 2)}(2 t)$ (see Eq. (5.32) of [Ko3]) which in particular implies $m_{1}^{(\alpha, \alpha)}(t)=\frac{1}{2} m_{1}^{(\alpha,-1 / 2)}(2 t)$. 
2.7 Remark. The preceding proof leads easily to explicit constants $C=C(\alpha, \beta)$ in Proposition 2.5. For instance, for $\alpha>\beta+1$, one may take $\varepsilon=1 / 3$ above and obtains from (2.6) and explicit values of beta-integrals that $A_{2}, A_{3} \leq \frac{6 \alpha}{e(\alpha-\beta-1)}$ and thus, by (2.5), that

$$
C:=\frac{6 \alpha}{e(\alpha-\beta-1)}
$$

is an admissable constant in the statement of Proposition 2.5.

We finally use the integral representation 2.4 of $m_{1}$ in order to estimate $m_{1}$. Weaker estimates for $m_{1}$ for general Chebli-Trimeche hypergroups on [0, [ are given in [Z2],[Z3]; see Proposition 7.3.23 of $[\mathrm{BH}]$. A better estimation is derived in $[\mathrm{G}]$.

2.8 Lemma. Let $\alpha \geq \beta \geq-1 / 2$ with $\alpha>-1 / 2$. Then there exists a constant $C=C(\alpha, \beta)$ with $t-C \leq m_{1}(t) \leq t$ for all $t \geq 0$.

Proof. Let $t \geq 0, \varphi \in[0, \pi]$ and $r \in[0,1]$. Then

$$
e^{t}(1-r) / 2 \leq\left|\operatorname{ch} t+r \cdot e^{i \varphi} \operatorname{sh} t\right| \leq \operatorname{ch} t+\operatorname{sh} t=e^{t} .
$$

We conclude from (2.4) that

$$
m_{1}(t)=\int_{0}^{1} \int_{0}^{\pi} \ln \left(\left|\operatorname{ch} t+r \cdot e^{i \varphi} \operatorname{sh} t\right|\right) d m_{\alpha, \beta} \leq \int_{0}^{1} \int_{0}^{\pi} \ln \left(e^{t}\right) d m_{\alpha, \beta}=t .
$$

For the second inequality, we now assume $\alpha>\beta$ and conclude from Lemma 2.6 for $\varepsilon=$ $(\alpha-\beta) / 2$ that $\ln (1-r) \geq-C /(1-r)^{\varepsilon}$ and thus

$$
m_{1}(t) \geq \int_{0}^{1} \int_{0}^{\pi} \ln \left(e^{t}(1-r) / 2\right) d m_{\alpha, \beta} \geq t-\ln 2-C \cdot \int_{0}^{1} \int_{0}^{\pi}(1-r)^{-\varepsilon} d m_{\alpha, \beta},
$$

where the latter integral has a finite value. This implies the result for $\alpha>\beta$. The case $\alpha=\beta>-1 / 2$ can again be handled as in the end of the proof of Proposition 2.5.

This lemma and Proposition 2.5 lead to:

2.9 Corollary. Let $\alpha \geq \beta \geq-1 / 2$ with $\alpha>-1 / 2$. Then there exists a constant $C=C(\alpha, \beta)$ such that for all $t \geq 0$ and $\lambda \in \mathbb{R}$,

$$
\left|\varphi_{i \rho-\lambda}^{(\alpha, \beta)}(t)-e^{i \lambda \cdot t}\right| \leq C\left(\lambda^{2}+|\lambda|^{3}\right) .
$$

\section{Central limit theorems for growing parameters}

In this section we derive two CLTs for Jacobi random walks, where in the first result $\alpha$ tends to infinity with fixed $\beta$, while in the second one $\alpha$ and $\beta$ tend to infinity.

3.1 Theorem. Let $\beta \geq-1 / 2$ fixed, and let $\left(\alpha_{n}\right)_{n \geq 1} \subset[\beta, \infty[$ be an increasing sequence of parameters with $\lim _{n \rightarrow \infty} n / \alpha_{n}=0$. Let $\nu \in M^{1}([0, \infty[)$ be a probability measure with a finite second moment $\int_{0}^{\infty} x^{2} d \nu(x)<\infty$ and with $\nu \neq \delta_{0}$, and consider the associated Jacobi random walks $\left(S_{n}^{\left(\alpha_{n}, \beta\right)}\right)_{n \geq 0}$ on $[0, \infty[$. Then

$$
\frac{S_{n}^{\left(\alpha_{n}, \beta\right)}-n \cdot m_{1}}{\sqrt{n}} \rightarrow N\left(0, m_{2}-m_{1}^{2}\right)
$$


in distribution for $n \rightarrow \infty$ with a normal distribution $N\left(0, m_{2}-m_{1}^{2}\right)$ with parameters

$$
\left.m_{1}:=\int_{0}^{\infty} \ln (\operatorname{ch} x) d \nu(x)<\infty, \quad m_{2}:=\int_{0}^{\infty}(\ln (\operatorname{ch} x))^{2} d \nu(x) \in\right] m_{1}^{2}, \infty[.
$$

Proof. Consider the homeomorphism $T:[0, \infty[\rightarrow[0, \infty[, \quad t \mapsto \ln \operatorname{ch} t$. We recapitulate from Proposition 2.2 that

$$
\varphi_{i \rho-\lambda}^{\left(\alpha_{n}, \beta\right)}(t)=e^{i \lambda \cdot \ln \operatorname{ch} t}+O\left(|\lambda| / \sqrt{\alpha}_{n}\right)
$$

uniformly in $t \in[0, \infty[$. Therefore, there exists a constant $C>0$ with

$$
\left|\int_{0}^{\infty} \varphi_{i \rho-\lambda}^{\left(\alpha_{n}, \beta\right)}(t) d \nu^{\left(n ; \alpha_{n}, \beta\right)}(t)-\int_{0}^{\infty} e^{i \lambda \cdot \ln \ln t} d \nu^{\left(n ; \alpha_{n}, \beta\right)}(t)\right| \leq C \cdot \frac{|\lambda|}{\sqrt{\alpha}_{n}}
$$

and

$$
\left|\int_{0}^{\infty} \varphi_{i \rho-\lambda}^{\left(\alpha_{n}, \beta\right)}(t) d \nu(t)-\int_{0}^{\infty} e^{i \lambda \cdot \ln \operatorname{ch} t} d \nu(t)\right| \leq C \cdot \frac{|\lambda|}{\sqrt{\alpha}_{n}}
$$

for $\lambda \in \mathbb{R}$ and $n \in \mathbb{N}$. Moreover, the random variables $T\left(S_{n}^{\left(\alpha_{n}, \beta\right)}\right)$ have the distributions $T\left(\nu^{\left(n ; \alpha_{n}, \beta\right)}\right)$ with the classical Fourier transforms

$$
T\left(\nu^{\left(n ; \alpha_{n}, \beta\right)}\right)^{\wedge}(\lambda)=\int_{0}^{\infty} e^{-i \lambda \cdot \ln \operatorname{ch} t} d \nu^{\left(n ; \alpha_{n}, \beta\right)}(t) .
$$

Therefore, by (3.1) and (3.2),

$$
\begin{aligned}
T\left(\nu^{\left(n ; \alpha_{n}, \beta\right)}\right)^{\wedge}(\lambda) & =\int_{0}^{\infty} \varphi_{i \rho+\lambda}^{\left(\alpha_{n}, \beta\right)}(t) d \nu^{\left(n ; \alpha_{n}, \beta\right)}(t)+O\left(|\lambda| / \sqrt{\alpha}_{n}\right) \\
& =\left(\int_{0}^{\infty} \varphi_{i \rho+\lambda}^{\left(\alpha_{n}, \beta\right)}(t) d \nu(t)\right)^{n}+O\left(|\lambda| / \sqrt{\alpha}_{n}\right) \\
& =\left(T(\nu)^{\wedge}(\lambda)+O\left(|\lambda| / \sqrt{\alpha}_{n}\right)\right)^{n}+O\left(|\lambda| / \sqrt{\alpha}_{n}\right) .
\end{aligned}
$$

Moreover, as $\nu$ has a finite second moment by our assumption, and as $\ln \operatorname{ch} t \leq t$ for $t \geq 0$, the measure $T(\nu)$ also has finite first and second moments

$$
m_{k}=\int_{0}^{\infty} t^{k} d T(\nu)(t)=\int_{0}^{\infty}(\ln \operatorname{ch} t)^{k} d \nu(t) \quad(k=1,2)
$$

and thus

$$
T(\nu)^{\wedge}(\lambda)=1-i \lambda m_{1}-\lambda^{2} m_{2} / 2+o\left(\lambda^{2}\right) \quad \text { for } \quad \lambda \rightarrow 0 .
$$

Therefore, if we denote the distribution of $\left(T\left(S_{n}^{\left(\alpha_{n}, \beta\right)}\right)-n \cdot m_{1}\right) / \sqrt{n}$ by $\mu_{n}$, and if we use the assumption $O\left(1 / \sqrt{n \alpha_{n}}\right)=o(1 / n)$, we conclude that for $\lambda \in \mathbb{R}$,

$$
\begin{aligned}
\mu_{n}^{\wedge}(\lambda) & =T\left(\nu^{\left(n ; \alpha_{n}, \beta\right)}\right)^{\wedge}(\lambda / \sqrt{n}) \cdot e^{i n \cdot m_{1} \lambda / \sqrt{n}} \\
& =\left(\left(T(\nu)^{\wedge}(\lambda / \sqrt{n})+O\left(|\lambda| / \sqrt{n \alpha_{n}}\right)\right)^{n}+O\left(|\lambda| / \sqrt{n \alpha_{n}}\right)\right) \cdot e^{i n \cdot m_{1} \lambda / \sqrt{n}} \\
& =\left(1-\frac{i \lambda m_{1}}{\sqrt{n}}-\frac{\lambda^{2} m_{2}}{2 n}+o(1 / n)+O\left(|\lambda| / \sqrt{n \alpha_{n}}\right)\right)^{n} \cdot\left(1+\frac{i \lambda m_{1}}{\sqrt{n}}-\frac{\lambda^{2} m_{1}^{2}}{2 n}+o(1 / n)\right)^{n} \\
& =\left(1-\frac{\lambda^{2}\left(m_{2}-m_{1}^{2}\right)}{2 n}+o(1 / n)\right)^{n},
\end{aligned}
$$


which tends for $n \rightarrow \infty$ to $e^{-\lambda^{2}\left(m_{2}-m_{1}^{2}\right) / 2}=N\left(0, m_{2}-m_{1}^{2}\right)^{\wedge}(\lambda)$. The classical continuity theorem of Levy yields that $\left(T\left(S_{n}^{\left(\alpha_{n}, \beta\right)}\right)-n m_{1}\right) / \sqrt{n}$ tends in distribution to $N\left(0, m_{2}-m_{1}^{2}\right)$. This in particular shows that $\ln \left(\operatorname{ch}\left(S_{n}^{\left(\alpha_{n}, \beta\right)}\right)\right) / n \rightarrow m_{1}>0$ and thus $e^{-2 S_{n}^{\left(\alpha_{n}, \beta\right)}} \rightarrow 0$ in probability. Using

$$
x-\ln 2 \leq \ln (\operatorname{ch} x) \leq x+\ln \left(1+e^{-2 x}\right) \leq x+e^{-2 x}
$$

and thus

$$
\ln \operatorname{ch} S_{n}^{\left(\alpha_{n}, \beta\right)}-e^{-2 S_{n}^{\left(\alpha_{n}, \beta\right)}} \leq S_{n}^{\left(\alpha_{n}, \beta\right)} \leq \ln \operatorname{ch} S_{n}^{\left(\alpha_{n}, \beta\right)}+\ln 2,
$$

we obtain that $\left(S_{n}^{\left(\alpha_{n}, \beta\right)}-n m_{1}\right) / \sqrt{n}$ tends in distribution to $N\left(0, m_{2}-m_{1}^{2}\right)$ as claimed.

3.2 Remark. The preceding theorem was derived in [V3] by completely different methods under the stronger condition $n / \sqrt{\alpha}_{n} \rightarrow 0$ for $n \rightarrow \infty$. Recently, the preceding theorem was generalized by W. Grundmann [G] to an arbitrary sequence $\left(\alpha_{n}\right)_{n}$ with $\alpha_{n} \rightarrow \infty$.

The following CLT can be proved in the same way as Theorem 3.1 by using Proposition 2.2 and the homeomorphism $T:\left[0, \infty\left[\rightarrow\left[0, \infty\left[\right.\right.\right.\right.$ with $T(x):=\ln \sqrt{\operatorname{ch}^{2} x-(1 / c) \operatorname{sh}^{2} x}$ instead of Proposition 2.1 and $T(x):=\ln \operatorname{ch} x$. We expect that it can also be generalized to an arbitrary sequence $\left(\alpha_{n}\right)_{n}$ with $\alpha_{n} \rightarrow \infty$ similar to [G].

3.3 Theorem. Fix constants $c>1$ and $d>0$, and let $\left(\beta_{n}\right)_{n \geq 1} \subset[\beta, \infty[$ be an increasing sequence of parameters with $\lim _{n \rightarrow \infty} n / \beta_{n}=0$. Moreover, put $\alpha_{n}:=c \beta_{n}+d$.

Let $\nu \in M^{1}\left(\left[0, \infty[)\right.\right.$ be a probability measure with a finite second moment $\int_{0}^{\infty} x^{2} d \nu(x)<\infty$ and with $\nu \neq \delta_{0}$, and consider the associated Jacobi random walks $\left(S_{n}^{\left(\alpha_{n}, \beta_{n}\right)}\right)_{n \geq 0}$ on $[0, \infty[$. Then

$$
\frac{S_{n}^{\left(\alpha_{n}, \beta_{n}\right)}-n \cdot m_{1}}{\sqrt{n}} \rightarrow N\left(0, m_{2}-m_{1}^{2}\right)
$$

in distribution for $n \rightarrow \infty$ with a normal distribution $N\left(0, m_{2}-m_{1}^{2}\right)$ with parameters

$$
\begin{gathered}
m_{1}:=\int_{0}^{\infty} \ln \sqrt{\operatorname{ch}^{2} x-(1 / c) \operatorname{sh}^{2} x} d \nu(x)>0 \\
\left.m_{2}:=\int_{0}^{\infty}\left(\ln \sqrt{\operatorname{ch}^{2} x-(1 / c) \operatorname{sh}^{2} x}\right)^{2} d \nu(x) \in\right] m_{1}^{2}, \infty[.
\end{gathered}
$$

\section{Central limit theorems for fixed parameters}

In this section we present a couple of CLTs for fixed parameters $\alpha, \beta$. We consider the following setting: We fix some non-trivial probability measure $\nu \in M^{1}\left(\left[0, \infty[)\right.\right.$ with $\nu \neq \delta_{0}$ which possibly satisfies some moment condition. For each $d \in] 0,1]$ consider the compressing map $D_{d}: x \mapsto d x$ on $\left[0, \infty\left[\right.\right.$ as well as the compressed measure $\nu_{d}:=D_{d}(\nu) \in M^{1}([0, \infty[)$. For given $\nu$ and $d$ we consider a Jacobi random walk $\left(S_{n}^{(\alpha, \beta, d)}\right)_{n \geq 0}$ on $[0, \infty[$ associated with the law $\nu_{d}$ as above. We now investigate the limit behavior of $\left(S_{n}^{\left(\alpha, \beta, n^{-r}\right)}\right)_{n \geq 0}$ for different powers $r \geq 0$. The most classical cases appear for $r=1 / 2$ and $r=0$.

In fact, for $r=1 / 2$, the random variables $S_{n}^{\left(\alpha, \beta, n^{-1 / 2}\right)}$ tend in distribution to some probability measure $\gamma_{t_{0}}^{(\alpha, \beta)}$ which is part of the unique (up to time parametrization) Gaussian convolution semigroup $\left(\gamma_{t}^{(\alpha, \beta)}\right)_{t \geq 0}$ on the Jacobi hypergroup on $\left[0, \infty\right.$ [ where the correct $t_{0}$ 
mainly depends on the second moment $m_{2}:=\int_{0}^{\infty} x^{2} d \nu(x)$ of $\nu$. For details see Theorem 7.4.1 of $[\mathrm{BH}]$. We remark that this CLT holds for general Chebli-Trimeche hypergroups on $[0, \infty[$. As the proof is very standard and universal, we do not treat this case here.

The case $r=0$ was handled by Zeuner [Z1] for Chebli-Trimeche hypergroups on [0, $\infty[$ with exponential growth by using an estimation weaker than Proposition 2.5. We here reprove this CLT together with a Berry-Esseen-type order of convergence $O\left(n^{-1 / 3}\right)$ which is slightly worse than the order $O\left(n^{-1 / 2}\right)$ in the classical CLT for sums of iid random variables on $\mathbb{R}$.

A CLT in the case $r>1 / 2$ was treated earlier in in Section 3 of [V2] on the basis of a variant of Proposition 2.4. We here derive this CLT on the basis of Proposition 2.4. The reason for doing so is, that in our opinion, the proof of Proposition 2.4 above is more elementary than the corresponding variant in Section 3 of [V2], and that the present proof can be transfered without problems to a higher dimensional setting.

We finally turn to the case $r \in] 0,1 / 2$ [ which was not handled before. It turns out that the cases $r \in] 0,1 / 6[, r=1 / 6$, and $r \in] 1 / 6,1 / 2[$ lead to a different limit behavior.

Before going into details, we collect some properties of the moment functions $m_{k}$ introduced in (2.4) from the literature.

4.1 Lemma. (1) $m_{1}$ is increasing on $[0, \infty[$.

(2) There is a constant $R>0$ with $m_{1}(x) \leq R x^{2}$ for all $x \geq 0$.

(3) For all $\mu, \nu \in M^{1}([0, \infty[)$ with finite first moment,

$$
\int_{0}^{\infty} m_{1} d(\mu * \nu)=\int_{0}^{\infty} m_{1} d \mu+\int_{0}^{\infty} m_{1} d \nu
$$

(4) For $k \in \mathbb{N}$ and $t \geq 0, m_{k}(x) \leq x^{k}$.

Proof. For (1), (3), and (4) see [Z2] or Section 7.2 of [BH]. Part (2) follows from the fact that $m_{1}$ is analytic with $m_{1}^{\prime}(0)=0$ in combination with Lemma 2.8 .

4.2 Theorem. Let $\nu \in M^{1}\left(\left[0, \infty[)\right.\right.$ with $\nu \neq \delta_{0}$ and finite second moment and $\left(S_{n}^{(\alpha, \beta, 1)}\right)_{n}$ the associated Jacobi random walk without initial compression. For $k \in \mathbb{N}$ let

$$
\left.\left.M_{k}:=\int_{0}^{\infty} m_{k} d \nu(t) \in\right] 0, \infty\right]
$$

be the so called modified moments of $\nu$ with $M_{1}, M_{2}<\infty$ by our assumption. Then

$$
\left(S_{n}^{(\alpha, \beta, 1)}-n M_{1}\right) / \sqrt{n}
$$

tends in distribution to $N\left(0, M_{2}-M_{1}^{2}\right)$.

Moreover, if $\nu \in M^{1}([0, \infty[)$ in addition has third moments, then the distribution functions of $\left(S_{n}^{(\alpha, \beta, 1)}-n M_{1}\right) / \sqrt{n}$ tend uniformly to the distribution function of $N\left(0, M_{2}-M_{1}^{2}\right)$ of order $n^{-1 / 3}$.

Proof. For the proof of the first statement on the basis of Corollary 2.9 we refer to Theorem 7.4 .2 of $[\mathrm{BH}]$.

For the second statement fix a small constant $c>0$ and put $T:=c n^{1 / 3}$. Moreover, let $n \in \mathbb{N}$ and $\lambda \in \mathbb{R}$ with $|\lambda| \leq T$. As $\nu$ has a finite third moment, the function $\tilde{\nu}: \mathbb{R} \rightarrow$ 
$\mathbb{C}, \lambda \mapsto \int_{0}^{\infty} \varphi_{i \rho-\lambda}^{(\alpha, \beta)}(t) d \nu(t)$ is three times differentiable (see [Z1] or 7.2.19 of [BH]), i.e., by the remainder in the Taylor expansion,

$$
\left|\tilde{\nu}(\lambda / \sqrt{n})-\left(1+i \lambda M_{1} / \sqrt{n}-\lambda^{2} M_{2} /(2 n)\right)\right| \leq \frac{\lambda^{3} M_{3}}{6 n^{3 / 2}}
$$

for $n \in \mathbb{N}$ and $\lambda \in \mathbb{R}$. Therefore, for suitable positive constants $C_{1}, C_{2}, \ldots$,

$$
\left|e^{-i \lambda M_{1} / \sqrt{n}} \tilde{\nu}(\lambda / \sqrt{n})-\left(1-\frac{\lambda^{2}}{2 n}\left(M_{2}-M_{1}^{2}\right)\right)\right| \leq C_{1} \frac{\lambda^{3}}{n^{3 / 2}},
$$

and in particular, for $|\lambda| \leq T$ and $c>0$ sufficiently small,

$$
|\tilde{\nu}(\lambda / \sqrt{n})| \leq 1-\frac{\lambda^{2}}{2 n}\left(M_{2}-M_{1}^{2}\right)+C_{1} \frac{|\lambda|^{3}}{n^{3 / 2}} \leq 1-\frac{C_{2} \lambda^{2}}{n} \leq e^{-C_{2} \lambda^{2} / n} .
$$

Moreover, under this restriction and by the same arguments,

$$
\left|e^{-\lambda^{2}\left(M_{2}-M_{1}^{2}\right) /(2 n)}-\left(1-\frac{\lambda^{2}}{2 n}\left(M_{2}-M_{1}^{2}\right)\right)\right| \leq C_{3} \frac{\lambda^{4}}{n^{2}}
$$

and $\left|e^{-\lambda^{2}\left(M_{2}-M_{1}^{2}\right) /(2 n)}\right| \leq e^{-C_{2} \lambda^{2} / n}$. Therefore, using $\left|a^{n}-b^{n}\right| \leq n|a-b| \cdot \max (|a|,|b|)^{n-1}$, we get

$$
\begin{aligned}
& \left|e^{-i \lambda M_{1} \sqrt{n}} \tilde{\nu}(\lambda / \sqrt{n})^{n}-e^{-\lambda^{2}\left(M_{2}-M_{1}^{2}\right) / 2}\right| \\
& \quad \leq n \cdot\left|e^{-i \lambda M_{1} / \sqrt{n}} \tilde{\nu}(\lambda / \sqrt{n})-e^{-\lambda^{2}\left(M_{2}-M_{1}^{2}\right) /(2 n)}\right| \cdot C_{4} \cdot e^{-C_{5} \lambda^{2}} \\
& \quad \leq C_{6} \cdot \frac{\lambda^{3}}{n^{1 / 2}} \cdot e^{-C_{5} \lambda^{2}} .
\end{aligned}
$$

On the other hand, by Corollary 2.9 and the multiplicativity of the Jacobi functions,

$$
\left.\tilde{\nu}(\lambda / \sqrt{n})^{n}=\int_{0}^{\infty} \varphi_{i \rho-\lambda}^{(\alpha, \beta)}(t) d \nu^{(} n\right)(t)=\int_{0}^{\infty} e^{i \lambda t} d \nu^{(n)}(t)+O\left(\lambda^{2}+|\lambda|^{3}\right) .
$$

Therefore, by (4.1), the usual Fourier transform $\widehat{\nu^{(n)}}$ of $\nu^{(n)}$ satisfies

$$
\begin{aligned}
& \int_{-T}^{T} \frac{\widehat{\mid \nu^{(n)}}(\lambda / \sqrt{n}) \cdot e^{i \lambda M_{1} \cdot \sqrt{n}}-e^{-\lambda^{2}\left(M_{2}-M_{1}^{2}\right) / 2} \mid}{|\lambda|} d \lambda \\
& \quad \leq C_{7} \int_{-T}^{T}\left(\frac{|\lambda|}{n}+\frac{\left|\lambda^{2}\right|}{n^{3 / 2}}+\frac{\lambda^{2}+|\lambda|^{3}}{n^{1 / 2}} \cdot e^{-C_{5} \lambda^{2}}\right) d \lambda
\end{aligned}
$$

As this expression is of order $O\left(n^{-1 / 3}\right)$ for $T=c n^{1-/ 3}$, we conclude from the lemma of Berry-Esseen (see, for instance, Lemma 2 in Section XVI.3 of Feller [Fe]) that the distribution functions of $\left(S_{n}^{(\alpha, \beta, 1)}-n M_{1}\right) / \sqrt{n}$ tend uniformly to the distribution function of $N\left(0, M_{2}-M_{1}^{2}\right)$ of order $n^{-1 / 3}$ as claimed.

4.3 Theorem. Let $\nu \in M^{1}\left(\left[0, \infty[)\right.\right.$ with $\nu \neq \delta_{0}$ and finite second moment $m_{2}:=\int_{0}^{\infty} x^{2} d \mu(x) \in$ ] $0, \infty[$. Let $\alpha \geq \beta \geq-1 / 2$ with $\alpha>-1 / 2$, and let $r>1 / 2$. Then

$$
\left(\frac{2(\alpha+1)}{m_{2}}\right)^{1 / 2} n^{r-1 / 2} \cdot S_{n}^{\left(\alpha, \beta, n^{-r}\right)}
$$


tends in distribution to the Rayleigh distribution $\rho_{\alpha}$ with Lebesgue density

$$
\frac{1}{2^{\alpha} \Gamma(\alpha+1)} x^{2 \alpha+1} e^{-x^{2} / 2} \quad(x \geq 0) .
$$

The proof needs some preparations. The following estimation is proved in Lemma 3.5 of [V2] by using Lemma 4.1

4.4 Lemma. There exists a constant $M=M(\alpha, \beta, r, \nu)>0$ such that

$$
\mathbf{P}\left(S_{n}^{\left(\alpha, \beta, n^{-r}\right)} \geq c\right) \leq \frac{M}{m_{1}(c) n^{2 r-1}} \quad \text { for } \quad c>0, n \in \mathbb{N}
$$

For the rest of the proof of Theorem 4.3 we denote the distribution of $n^{r-1 / 2} S_{n}^{\left(\alpha, \beta, n^{-r}\right)}$ by $\mu_{n}$. The proofs of the following two lemmas are similar to, but slightly different from those of Lemmas 3.6 and 3.7 of [V2].

4.5 Lemma. For all $\lambda \geq 0$,

$$
\lim _{n \rightarrow \infty} \int_{0}^{\infty}\left|j_{\alpha}(\lambda t)-\varphi_{\lambda n^{r-1 / 2}}^{(\alpha, \beta)}\left(n^{1 / 2-r}\right)\right| d \mu_{n}(t)=0
$$

Proof. Let $c>0$. Then, by Proposition 2.4, the boundedness of the involved Jacobi and Bessel functions and by the preceding lemma,

$$
\begin{gathered}
A_{n}:=\int_{0}^{\infty}\left|j_{\alpha}(\lambda t)-\varphi_{\lambda n^{r-1 / 2}}^{(\alpha, \beta)}\left(t n^{1 / 2-r}\right)\right| d \mu_{n}(t)=\left(\int_{0}^{c}+\int_{c}^{\infty}\right)|\ldots| d \mu_{n}(t) \\
\leq \frac{H(c)|\lambda|}{n^{r-1 / 2}}+2 \cdot \mathbf{P}\left(n^{r-1 / 2} S_{n}^{\left(\alpha, \beta, n^{-r}\right)} \geq c\right) \\
\leq \frac{H(c)|\lambda|}{n^{r-1 / 2}}+\frac{2 M}{m_{1}\left(c n^{1 / 2-r}\right) \cdot n^{2 r-1}}
\end{gathered}
$$

for some constants $M$ and some $H(c)$ depending on $c$. On the other hand, as $m_{1}^{\prime \prime}(0)>0$, there exist $a, b>0$ with $m_{1}(x) \geq a x^{2}$ for $x \in[0, b]$.

Now let $\varepsilon>0$. Then choose $c$ with $2 M /\left(a c^{2}\right) \leq \varepsilon / 2$, and now $n$ large enough with

$$
\frac{H(c)|\lambda|}{n^{r-1 / 2}} \leq \varepsilon / 2 \quad \text { and } \quad c n^{1 / 2-r} \leq b
$$

As the latter implies

$$
\frac{2 M}{m_{1}\left(c n^{1 / 2-r}\right) \cdot n^{2 r-1}} \leq \frac{2 M}{a c^{2}} \leq \varepsilon / 2
$$

we obtain from (4.3) that $A_{n} \leq \varepsilon$ for large $n$ as claimed.

4.6 Lemma. Let $\lambda>0$ and $\nu \in M^{1}\left(\left[0, \infty[)\right.\right.$ with finite second moment $m_{2}<\infty$. Then, for $n \rightarrow \infty$,

$$
\int_{0}^{\infty} \varphi_{\lambda n^{r-1 / 2}}^{(\alpha, \beta)}\left(t / n^{r}\right) d \nu(t)=1-\frac{\lambda^{2} m_{2}}{4(\alpha+1) n}+o(1 / n)
$$


Proof. Consider $H_{\lambda}(t):=\left|\varphi_{\lambda}^{(\alpha, \beta)}(t)-j_{\alpha}(\lambda t)\right| \leq 2$, We apply Proposition 2.4 to $t / n^{r} \leq 1$ instead of $t$ with $n=1$ there. Therefore, for some $C>1$, and by Markov's inequality,

$$
\begin{aligned}
\int_{0}^{\infty} H_{\lambda n^{r-1 / 2}}\left(t / n^{r}\right) d \nu(t) & \leq \int_{0}^{n^{r}} H_{\lambda n^{r-1 / 2}}\left(t / k^{r}\right) d \nu(t)+2 \nu\left(\left[n^{r}, \infty[)\right.\right. \\
& \leq C \lambda \cdot \int_{0}^{n^{r}} \frac{t^{2} n^{r-1 / 2}}{n^{2 r}} d \nu(t)+2 \nu\left(\left[n^{r}, \infty[)\right.\right. \\
& \leq C \lambda m_{2} / n^{r+1 / 2}+2 m_{2} / n^{2 r}=o(1 / n) .
\end{aligned}
$$

Furthermore, as $m_{2}<\infty$, the Hankel transform $g(\lambda):=\int_{0}^{\infty} j_{\alpha}(\lambda t) d \nu(t)$ of $\nu$ is two-times differentiable at $\lambda=0$ with

$$
g(\lambda)=1-\frac{\lambda^{2} m_{2}}{4(\alpha+1)}+o\left(\lambda^{2}\right) \quad \text { for } \quad \lambda \rightarrow 0
$$

(see Theorem 4.7 of $[\mathrm{Z} 1]$ or Section 7.2 of $[\mathrm{BH}]$ ) with $m_{1}=0$ and $m_{2}(x)=x^{2} /(\alpha+1)$ there). Hence, by (4.4),

$$
\begin{aligned}
\int_{0}^{\infty} \varphi_{\lambda n^{r-1 / 2}}^{(\alpha, \beta)}\left(t / n^{r}\right) d \mu(t) & =\int_{0}^{\infty} j_{\alpha}\left(\lambda t / n^{1 / 2}\right) d \mu(t)+o(1 / n) \\
& =1-\frac{x^{2} \lambda^{2}}{4(\alpha+1) n}+o(1 / n)
\end{aligned}
$$

as claimed.

Proof of Theorem 4.3. Fix $\lambda \in[0, \infty[$. Then Lemmas 4.5 and 4.6 lead to

$$
\begin{aligned}
\lim _{n \rightarrow \infty} & \int_{0}^{\infty} j_{\alpha}(\lambda t) d \mu_{n}(t)=\lim _{n \rightarrow \infty} \int_{0}^{\infty} \varphi_{x n^{r-1 / 2}}^{(\alpha, \beta)}\left(t n^{1 / 2-r}\right) d \mu_{n}(t) \\
& =\lim _{n \rightarrow \infty} \int_{0}^{\infty} \varphi_{\lambda n^{r-1 / 2}}^{(\alpha, \beta)}(t) d \nu_{n^{-r}}^{(n)}(t)=\lim _{n \rightarrow \infty}\left(\int_{0}^{\infty} \varphi_{\lambda n^{r-1 / 2}}^{(\alpha, \beta)}(t) d \nu_{n^{-r}}(t)\right)^{n} \\
& =\lim _{n \rightarrow \infty}\left(\int_{0}^{\infty} \varphi_{\lambda n^{r-1 / 2}}^{(\alpha, \beta)}\left(t / n^{r}\right) d \nu(t)\right)^{n}=\lim _{n \rightarrow \infty}\left(1-\frac{x^{2} m_{2}}{4(\alpha+1) n}+o(1 / n)\right)^{n} \\
& =e^{-\lambda^{2} m_{2} / 4(\alpha+1)}
\end{aligned}
$$

As the Rayleigh distribution $\rho_{\alpha}$ satisfies

$$
\int_{0}^{\infty} j_{\alpha}(\lambda t) d \rho_{\alpha}(t)=e^{-\lambda^{2} / 2}
$$

Levy's continuity theorem for the Hankel transform (see, for instance, Section 4.2 of $[\mathrm{BH}]$ ) now yields that $\left(\frac{2(\alpha+1)}{m_{2}}\right)^{1 / 2} n^{r-1 / 2} \cdot S_{n}^{\left(\alpha, \beta, n^{-r}\right)}$ tends to $\rho_{\alpha}$ as claimed.

4.7 Theorem. Let $\nu \in M^{1}\left(\left[0, \infty[)\right.\right.$ with $\nu \neq \delta_{0}$, with compact support, and thus with finite moments $\left.m_{k}:=\int_{0}^{\infty} x^{k} d \mu(x) \in\right] 0, \infty[(k \in \mathbb{N})$. Let $\alpha \geq \beta \geq-1 / 2$ with $\alpha>-1 / 2$ and $r \in] 0,1 / 2\left[\right.$. Then the random variables $S_{n}^{\left(\alpha, \beta, n^{-r}\right)}$ have the following behavior for $n \rightarrow \infty$. 
(1) If $r \in] 1 / 6,1 / 2[$, then

$$
\frac{S_{n}^{\left(\alpha, \beta, n^{-r}\right)}-\frac{\rho m_{2}}{2(\alpha+1)} \cdot n^{1-2 r}}{n^{1 / 2-r}}
$$

tends in distribution to $N\left(0, \frac{m_{2}}{2(\alpha+1)}\right)$.

(2) If $r=1 / 6$, then

$$
\frac{S_{n}^{\left(\alpha, \beta, n^{-1 / 6}\right)}-\frac{\rho m_{2}}{2(\alpha+1)} \cdot n^{2 / 3}}{n^{1 / 3}}
$$

tends in distribution to $N\left(-\frac{\rho(\alpha+3 \beta+2) m_{4}}{12(\alpha+1)(\alpha+2)}, \frac{m_{2}}{2(\alpha+1)}\right)$.

(3) If $r \in] 0,1 / 6[$, then

$$
\frac{S_{n}^{\left(\alpha, \beta, n^{-r}\right)}-\frac{\rho m_{2}}{2(\alpha+1)} \cdot n^{1-2 r}}{n^{1-4 r}} \longrightarrow-\frac{\rho(\alpha+3 \beta+2) m_{4}}{12(\alpha+1)(\alpha+2)}
$$

in probability.

Notice that the drift term $-\frac{\rho(\alpha+3 \beta+2) m_{4}}{12(\alpha+1)(\alpha+2)}$ in (2) and (3) is negative, and that the case (2) combines the features of the cases (1) and (3).

The proof will be based on a simple Taylor-type expansion of of the $\varphi_{\lambda}^{(\alpha, \beta)}$ which is an immediate consequence of the well-known representation of $\varphi_{\lambda}^{(\alpha, \beta)}$ as hypergeometric series.

4.8 Lemma. Let $\alpha \geq \beta \geq-1 / 2$ with $\alpha>-1 / 2$, and let $\lambda \in \mathbb{R}$ and $t \geq 0$. Then, for $a, r>0$ and $n \rightarrow \infty$,

$$
\begin{aligned}
\varphi_{i \rho-\lambda / n^{a}}^{(\alpha, \beta)}\left(t / n^{r}\right)= & 1+\frac{i \rho \lambda t^{2}}{2(\alpha+1) n^{a+2 r}}-\frac{\lambda^{2} t^{2}}{4(\alpha+1) n^{2 a+2 r}} \\
& -\frac{i \rho(\alpha+3 \beta+2) t^{4} \lambda}{12(\alpha+1)(\alpha+2) n^{a+4 r}}+O\left(n^{-a-6 r}\right)+O\left(n^{-2 a-4 r}\right)
\end{aligned}
$$

locally uniformly in $t \in[0, \infty[$.

Proof. Using $\rho=\alpha+\beta+1$ and Eq. (2.4) of [Ko3], we have

$$
\begin{aligned}
\varphi_{i \rho-\lambda / n^{a}}^{(\alpha, \beta)}\left(t / n^{r}\right)= & { }_{2} F_{1}\left(\rho+i \lambda /\left(2 n^{a}\right),-i \lambda /\left(2 n^{a}\right) ; \alpha+1 ;-\operatorname{sh}^{2}\left(t / n^{r}\right)\right) \\
= & 1+\frac{\left(\rho+i \lambda /\left(2 n^{a}\right)\right) \cdot i \lambda}{2 n^{a} \cdot(\alpha+1)} \cdot \operatorname{sh}^{2}\left(t / n^{r}\right) \\
& -\frac{\left(\rho+i \lambda /\left(2 n^{a}\right)\right)\left(\rho+1+i \lambda /\left(2 n^{a}\right)\right) i \lambda\left(1-i \lambda /\left(2 n^{a}\right)\right)}{4 n^{a} \cdot(\alpha+1)(\alpha+2)} \cdot \operatorname{sh}^{4}\left(t / n^{r}\right) \\
& +O\left(n^{-a-6 r}\right) \\
= & 1+\frac{\left(\rho+i \lambda /\left(2 n^{a}\right)\right) \cdot i \lambda}{2 n^{a} \cdot(\alpha+1)} \cdot\left(\frac{t}{n^{r}}+\frac{t^{3}}{6 n^{3 r}}\right)^{2} \\
& -\frac{\left(\rho+i \lambda /\left(2 n^{a}\right)\right)\left(\rho+1+i \lambda /\left(2 n^{a}\right)\right) i \lambda\left(1-i \lambda /\left(2 n^{a}\right)\right)}{4 n^{a} \cdot(\alpha+1)(\alpha+2)} \cdot \frac{t^{4}}{n^{4 r}} \\
& +O\left(n^{-a-6 r}\right)+O\left(n^{-2 a-4 r}\right)
\end{aligned}
$$

locally uniformly in $t$ which readily leads to the claim. 
This expansion leads immediately to:

4.9 Corollary. Let $\nu \in M^{1}([0, \infty[)$ with compact support. Then, in the setting of the preceding lemma,

$$
\begin{aligned}
\int_{0}^{\infty} \varphi_{i \rho-\lambda / n^{a}}^{(\alpha, \beta)}\left(t / n^{r}\right) d \nu(t)=1 & +\frac{i \rho \lambda m_{2}}{2(\alpha+1) n^{a+2 r}}-\frac{\lambda^{2} m_{2}}{4(\alpha+1) n^{2 a+2 r}} \\
& -\frac{i \rho(\alpha+3 \beta+2) m_{4} \lambda}{12(\alpha+1)(\alpha+2) n^{a+4 r}}+O\left(n^{-a-6 r}\right)+O\left(n^{-2 a-4 r}\right) .
\end{aligned}
$$

Proof of Theorem 4.7. We first recapitulate for all cases that by Corollary 2.9,

$$
\left|\varphi_{i \rho-\lambda}^{(\alpha, \beta)}(t)-e^{i \lambda \cdot t}\right|=O\left(\lambda^{2}+|\lambda|^{3}\right)
$$

uniformly in $t$. We now consider the different cases:

(1) Let $r \in] 1 / 6,1 / 2[$. In this case we put $a:=1 / 2-r \in] 0,1 / 3[$ and observe that, due to $r \geq 1 / 6,1=2 a+2 r<a+4 r$. Therefore, Eq. (4.7), the definition of the measures $\nu_{n^{-r}}$ above, the multiplicativity of Jacobi functions, and the preceding corollary imply that for all $\lambda \in \mathbb{R}$ and $n \in \mathbb{N}$, the classical Fourier transform of the distribution $\mu_{n}:=\nu_{n^{-r}}^{(n)}$ of the random variable $S_{n}^{\left(\alpha, \beta, n^{-r}\right)}$ satisfies

$$
\begin{aligned}
\hat{\mu}_{n}\left(\lambda / n^{a}\right) & =\int_{0}^{\infty} e^{-i t \lambda / n^{a}} d \nu_{n^{-r}}^{(n)}(t) \\
& =\int_{0}^{\infty} \varphi_{i \rho+\lambda / n^{a}}^{(\alpha, \beta)}(t) d \nu_{n^{-r}}^{(n)}(t)+o(1) \\
& =\left(\int_{0}^{\infty} \varphi_{i \rho+\lambda / n^{a}}^{(\alpha, \beta)}(t) d \nu_{n^{-r}}(t)\right)^{n}+o(1) \\
& =\left(\int_{0}^{\infty} \varphi_{i \rho+\lambda / n^{a}}^{(\alpha, \beta)}\left(t / n^{r}\right) d \nu(t)\right)^{n}+o(1) \\
& =\left(1-\frac{i \rho \lambda m_{2}}{2(\alpha+1) n^{a+2 r}}-\frac{\lambda^{2} m_{2}}{4(\alpha+1) n}+o(1 / n)\right)^{n}+o(1) .
\end{aligned}
$$

Therefore, using $a=1 / 2-r$,

$$
\begin{aligned}
\hat{\mu}_{n}\left(\lambda / n^{a}\right) \cdot \exp \left(\frac{i \rho m_{2} \lambda n^{1 / 2-r}}{2(\alpha+1)}\right) \\
=\left(1-\frac{i \rho \lambda m_{2}}{2(\alpha+1) n^{1 / 2+r}}-\frac{\lambda^{2} m_{2}}{4(\alpha+1) n}+o(1 / n)\right)^{n} . \\
\quad \cdot\left(1+\frac{i \rho \lambda m_{2}}{2(\alpha+1) n^{1 / 2+r}}+O\left(n^{-1-2 r}\right)\right)^{n}+o(1) \\
=\left(1-\frac{\lambda^{2} m_{2}}{4(\alpha+1) n}+o(1 / n)\right)^{n}+o(1)
\end{aligned}
$$

which tends for $\lambda \in \mathbb{R}$ to $\exp \left(-\lambda^{2} m_{2} /(4(\alpha+1))\right.$. Levy's continuity theorem for the classical Fourier transform now implies that

$$
\frac{S_{n}^{\left(\alpha, \beta, n^{-r}\right)}-\frac{\rho m_{2}}{2(\alpha+1)} \cdot n^{1-2 r}}{n^{1 / 2-r}}
$$

tends in distribution to $N\left(0, \frac{m_{2}}{2(\alpha+1)}\right)$ as claimed. 
(2) Now let $r=1 / 6$. Here we put $a:=1 / 6$. Then $2 a+2 r=a+4 r=1$ and $a+2 r=2 / 3$. As in the first case, we obtain from the preceding corollary

$$
\begin{aligned}
\hat{\mu}_{n}\left(\lambda / n^{1 / 3}\right) \cdot \exp \left(\frac{i \rho m_{2} \lambda n^{1 / 3}}{2(\alpha+1)}\right) \\
=\left(1-\frac{i \rho \lambda m_{2}}{2(\alpha+1) n^{2 / 3}}-\frac{\lambda^{2} m_{2}}{4(\alpha+1) n}+\frac{i \rho(\alpha+3 \beta+2) \lambda m_{4}}{12(\alpha+1)(\alpha+2) n}+o(1 / n)\right)^{n} . \\
\quad \cdot\left(1+\frac{i \rho \lambda m_{2}}{2(\alpha+1) n^{2 / 3}}+O\left(n^{-4 / 3}\right)\right)^{n}+o(1)
\end{aligned}
$$

which tends for $\lambda \in \mathbb{R}$ to $\exp \left(-\frac{\lambda^{2} m_{2}}{4(\alpha+1)}+\frac{i \rho(\alpha+3 \beta+2) \lambda m_{4}}{12(\alpha+1)(\alpha+2)}\right)$. The proof is now completed as before.

(3) Let $r \in] 0,1 / 6[$. We here put $a:=1-4 r \in] 1 / 3,1[$, and obtain $2 a+2 r>a+4 r=1$ and $a+2 r=1-2 r$. As in the first and second part, we obtain

$$
\begin{aligned}
\hat{\mu}_{n}\left(\lambda / n^{a}\right) \cdot \exp \left(\frac{i \rho m_{2} \lambda n^{2 r}}{2(\alpha+1)}\right) \\
=\left(1-\frac{i \rho \lambda m_{2}}{2(\alpha+1) n^{1-2 r}}+\frac{i \rho(\alpha+3 \beta+2) \lambda m_{4}}{12(\alpha+1)(\alpha+2) n}+o(1 / n)\right)^{n} . \\
\quad \cdot\left(1+\frac{i \rho \lambda m_{2}}{2(\alpha+1) n^{1-2 r}}+O\left(n^{-2+4 r}\right)\right)^{n}+o(1)
\end{aligned}
$$

which tends for $\lambda \in \mathbb{R}$ to $\exp \left(\frac{i \rho(\alpha+3 \beta+2) \lambda m_{4}}{12(\alpha+1)(\alpha+2)}\right)$. Therefore, again by Levy's continuity theorem,

$$
\frac{S_{n}^{\left(\alpha, \beta, n^{-r}\right)}-\frac{\rho m_{2}}{2(\alpha+1)} \cdot n^{1-2 r}}{n^{1-4 r}} \longrightarrow-\frac{\rho(\alpha+3 \beta+2) m_{4}}{12(\alpha+1)(\alpha+2)}
$$

in distribution and thus in probability.

\section{References}

[BH] W.R. Bloom, H. Heyer, Harmonic analysis of probability measures on hypergroups. De Gruyter Studies in Mathematics 20, de Gruyter-Verlag Berlin, New York 1995.

[F] J. Faraut, Analyse harmonique sur les pairs de Gelfand et les espaces hyperboliques. In: J.-L. Clerc et al.. Analyse Harmonique, C.I.M.P.A., Nice 1982, Ch. IV.

[Fe] W. Feller, An Introduction to Probability Theory and its Applications II. Wiley 1971.

[Ga] L. Gallardo, Comportement asymptotique des marches aleatoires associees aux polynomes de Gegenbauer. Adv. Appl. Prob. 16, 293 - 323 (1984).

[Gr] P. Graczyk, Dispersions and a central limit theorem on symmetric spaces. Bull. Sci. Math., II. Sr., 118, 105-116 (1994).

[G] W. Grundmann, Moment functions and central limit theorems for Jacobi hypergroups on $[0, \infty[$, Preprint, TU Dortmund, 2011. 
[H] G. Heckman, Dunkl Operators. Séminaire Bourbaki 828, 1996-97; Astérisque 245, 223-246 (1997).

[HS] G. Heckman, H. Schlichtkrull, Harmonic Analysis and Special Functions on Symmetric Spaces; Perspectives in Mathematics, vol. 16, Academic Press, California, 1994.

[Hel] S. Helgason, Groups and Geometric Analysis. Mathematical Surveys and Monographs, vol. 83, AMS 2000.

[KTS] F.I. Karpelevich, V.N. Tutubalin, M.G. Shur, Limit theorems for the compositions of distributions in the Lobachevski plane and space. Theory Probab. Appl. 4, 399-402 (1959).

[Ko1] T. Koornwinder, The addition formula for Jacobi polynomials II. The Laplace type integral representation and the product formula. Report TW 133/72. Math. Centrum, Amsterdam 1972.

[Ko2] T. Koornwinder, Jacobi functions and analysis on noncompact semisimple Lie groups. In: Special Functions: Group Theoretical Aspects and Applications, Eds. Richard Askey et al., D. Reidel, Dordrecht-Boston-Lancaster, 1984.

[Ko3] T. Koornwinder, Jacobi polynomials of type BC, Jack polynomials, limit transitions and $O(\infty)$. Contemp. Math. 190, 283-286 (1995).

[O] E.M. Opdam, Lecture Notes on Dunkl Operators for Real and Complex Reflection Groups. MSJ Memoirs, vol. 8, Mathematical Society of Japan, Tokyo, 2000.

[R] M. Rösler, Positive convolution structure for a class of Heckman-Opdam hypergeometric functions of type BC. J. Funct. Anal. 258, 2779-2800 (2010).

[RV1] M. Rösler, M. Voit, Dunkl theory, convolution algebras, and related Markov processes. In: P. Graczyk et al., Harmonic and Stochastic Analysis of Dunkl Processes, Hermann, Paris, 2008, pp. 1-112.

[RV2] M. Rösler, M. Voit, Harish-Chandra integral and asymptotic results for some Heckman-Opdam hypergeometric functions of type BC. Preprint 2011.

[Sa] P. Sawyer, Spherical functions on $S O_{0}(p, q) / S O(p) \times S O(q)$. Canad. Math. Bull. 42, 486-498 (1999).

[ST] R.J. Stanton, P.A. Tomas, Expansions for spherical functions on noncompact symmetric spaces. Acta Math. 140, 251-271 (1978).

[Sz] G. Szegö, Orthogonal Polynomials. Am. Math. Soc. Coll. Publ. 23. Providence, R. I.: Amer. Math. Soc. 1959.

[Te] A. Terras, Harmonic Analysis on Symmetric Spaces and Applications I. SpringerVerlag 1985.

[Tr] K. Trimeche, Probabilites indefiniment divisibles et theoreme de la limite centrale pour une convolution generalissee sur la demi-droite. Seminaire d'Analyse Harmonique, Faculte des Sciences de Tunis, Dep. Math., 1976.

[Tu] V.N. Tutubalin, On the limit behaviour of compositions of measures in the plane and space of Lobachevski. Theory Probab. Appl. 7, 189-196 (1962).

[V1] M. Voit, Central limit theorems for a class of polynomial hypergroups. Adv. Appl. Prob. 22, 68-87 (1990).

[V2] M. Voit, Central limit theorems for Jacobi hypergroups. In: W.C. Connett (ed.) et al., Applications of hypergroups and related measure algebras. AMS, Seattle, 1993. Contemp. Math. 183, 395-411 (1995). 
[V3] M. Voit, Limit theorems for radial random walks on homogeneous spaces with growing dimensions. In: J. Hilgert, Joachim (ed.) et al., Proc. symp. on infinite dimensional harmonic analysis $I V$. On the interplay between representation theory, random matrices, special functions, and probability. Tokyo, World Scientific. 308-326 (2009).

[Z1] H. Zeuner, The central limit theorem for Chebli-Trimeche hypergroups. J. Theor. Probab. 2, 51-63 (1989).

[Z2] H. Zeuner, Laws of large numbers for hypergroups on $\mathbb{R}_{+}$. Math. Ann. 283, $657-678$ (1989).

[Z3] H. Zeuner, Moment functions and laws of large numbers on hypergroups. Math. Z. 211, 369-407 (1992). 